\title{
Obscure Gastrointestinal Haemorrhage from a Leiomyoblastoma
}

We present here the case of a 48 -year-old man who presented with collapse, secondary to hypovolaemia. There was no history of gastrointestinal blood loss, and no visible evidence of haematemesis or melaena on examination.

A year previously, he had undergone coronary artery bypass grafting for severe ischaemic heart disease, and was receiving aspirin $75 \mathrm{mg}$ daily after the surgery. Six weeks later, he presented with an acute upper gastrointestinal bleed, and at endoscopy was shown to have a chronic gastric ulcer in the mid-body of the stomach. The ulcer was benign, and was associated with Helicobacter pylori. The aspirin treatment was stopped, and eradication therapy was completed and confirmed by a $\mathrm{C} 13$ urea breath test. Healing of the ulcer was confirmed at a subsequent endoscopy eight weeks later, and a small leiomyoma was observed adjacent to the healed ulcer (Figure 1).

A repeat endoscopy after resuscitation showed that the leiomyoma was present as before, but there was no active bleeding. A mesenteric angiogram showed an abnormal circulation in the region of the left gastric artery. A laparotomy was carried out, and the patient was found to have a large haemorrhagic tumour arising from the anterior wall of the stomach. Part of the tumour was necrotic, and it had bled into the peritoneal cavity (Figure 2). Histology showed that it was a leiomyoblastoma.

Intraperitoneal bleeding from smooth-muscle tumours of the stomach is a rare cause of obscure gastrointestinal bleeding, and should be considered in any patient who is known to have such pathology. Leiomyoblastomas are a rare variant of this type of

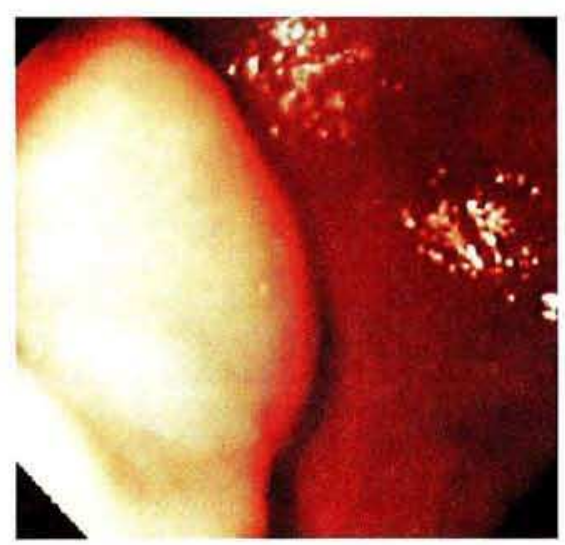

Figure 1: A small lesion with a central scar, apparently a leiomyoma, seen at endoscopy. 


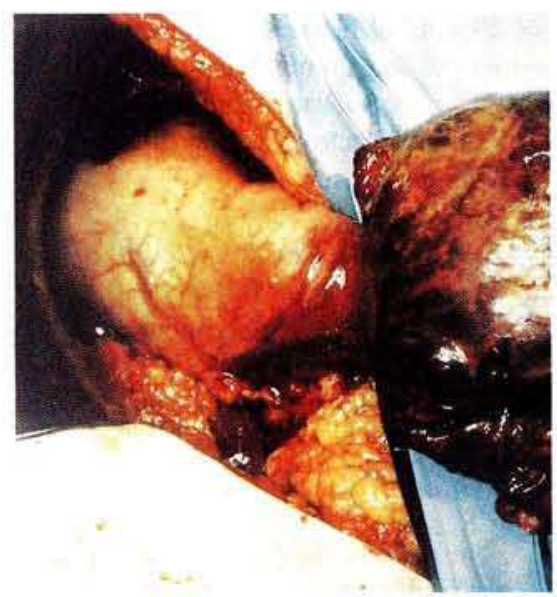

Figure 2: At laparotomy, a large leiomyoblastoma was found on the anterior wall of the stomach.

tumour (1), and the presentation usually involves chronic mild anaemia. Severe acute bleeding has not previously been described, and preoperative diagnosis of these silent tumours is often difficult. Large tumours have a $10-12 \%$ potential for malignancy $(2,3)$. Intraperitoneal bleeding should be considered in patients known to have leiomyomas, and angiography should be performed to locate the source of the bleeding.

A. Francis', T. Iqbal' ${ }^{2}$ I. M. Chesner ${ }^{2}$

' Dept. of Surgery

${ }^{2}$ Dept. of Gastroenterology,

Birmingham Heartlands Hospital,

Birmingham, United Kingdom

\section{References}

1. Waldrep D, Morgenstern L, Alshak NS. The enigma of leiomyoblastoma: spectrum of a rare gastric tumor. Am Surg 1993; 59: 256-60.

2. Plantiga ERM, Mravunac M, Joosten HJM. Gastric leiomyoblastoma: three interesting cases. Acta Chir Scan 1979; 145: $571-4$.

3. Appleman HD, Hewig EB. Gastric epithelial leiomyoma and leiomyosarcoma (leiomyoblastoma). Cancer 1976; 38: $708-28$.

Corresponding Author

I. M. Chesner, M.D., F.R.C.P.

Dept. of Gastroenterology

Birmingham Heartlands Hospital

Bordesley Green East

East Birmingham B95SS

United Kingdom

Fax: +44-121-771-2251 